TITLE:

\title{
Negative group delay and superluminal propagation: An electronic circuit approach
}

$\operatorname{AUTHOR}(\mathrm{S})$ :

Kitano, M; Nakanishi, T; Sugiyama, K

\section{CITATION:}

Kitano, M ... [et al]. Negative group delay and superluminal propagation: An electronic circuit approach. IEEE JOURNAL OF SELECTED TOPICS IN QUANTUM ELECTRONICS 2003, 9(1): 43-51

\section{ISSUE DATE:}

2003

URL:

http://hdl.handle.net/2433/39936

\section{RIGHT:}

(c)2003 IEEE. Personal use of this material is permitted. However, permission to reprint/republish this material for advertising or promotional purposes or for creating new collective works for resale or redistribution to servers or lists, or to reuse any copyrighted component of this work in other works must be obtained from the IEEE. 


\title{
Negative Group Delay and Superluminal Propagation: An Electronic Circuit Approach
}

\author{
Masao Kitano, Toshihiro Nakanishi, and Kazuhiko Sugiyama
}

\begin{abstract}
We present a simple electronic circuit which provides negative group delays for band-limited baseband pulses. It is shown that large time advancement comparable to the pulsewidth can be achieved with appropriate cascading of negative-delay circuits but eventually the out-of-band gain limits the number of cascading. The relations to superluminality and causality are also discussed.
\end{abstract}

Index Terms-Causality, filter, group velocity, negative group delay, superluminal propagation.

\section{INTRODUCTION}

B RILLOUIN AND Sommerfeld showed that in the region of anomalous dispersion, which is inside of the absorption band, the group velocity can exceed $c$, the light speed in a vacuum, or even be negative [1], [2]. Recently, it was shown that for a gain medium, superluminal propagation is possible at the outside of the gain resonance. Superluminal effects are also predicted in terms of quantum tunneling or evanescent waves [3]-[5]. Superluminal group velocities have been confirmed experimentally in various systems, and most controversies over this counterintuitive phenomenon have settled down. However, several questions seem to remain open; for example, "how far can we speed up the wave packets," "is it really nothing to do with information transmission," "what kind of applications are possible," and so on. In this paper, we will try to solve some of these problems by utilizing a simple circuit model for negative group delays.

Negative delay in lumped systems such as electronic circuits is very helpful to understanding various aspects of superluminal group velocity. Mitchell and Chiao [6], [7] constructed a bandpass amplifier with an inductance-capacitance $(L C)$ resonator and an operational amplifier. An arbitrary waveform generator is used to generate a Gaussian pulse by which a carrier is modulated. The circuit basically emulates an optical gain medium which shows anomalous dispersion in off-resonant region. Wang et al. [8] extended this circuit by using two $L C$ resonators which correspond to the two Raman gain lines [9], [10]. At the middle of two gain peaks, the frequency dependence of amplitude response is compensated and the pulse distortion can be minimized.

Manuscript received September 18, 2002. This work was supported in part by the Ministry of Education, Culture, Sports, Science and Technology in Japan under a Grant-in-Aid for Scientific Research 11216203 and by the National Science Foundation.

The authors are with the Department of Electronic Science and Engineering, Kyoto University, Kyoto 606-8501, Japan (e-mail: kitano@kuee.kyoto-u.ac.jp; t-naka@giga.kuee.kyoto-u.ac.jp; sugiyama@kuee.kyoto-u.ac.jp).

Digital Object Identifier 10.1109/JSTQE.2002.807979
The present authors [11] used an operational amplifier with an $R C$ feedback circuit. It provides negative delays for baseband pulses. In previous experiments, optical or electronic, a carrier frequency $\left(\omega_{0}\right)$ is modulated by a pulse which varies slowly compared with the carrier oscillation and the displacement of the envelopes is measured. Without carriers $\left(\omega_{0}=0\right)$, the system becomes much more simple. The amplitude response symmetric with respect to zero frequency is helpful to reduce the distortion. The baseband pulse is simply derived from a rectangular pulse generator and a series of low-pass filters.

The time constants can easily be set at the order of seconds and we can actually observe that the output light-emitting diode (LED) is lit earlier than the input LED. In addition to the usefulness as a demonstration tool, this circuit turned out to be very convenient to look into the essentials of negative group delays and superluminal propagation because of its simplicity.

In this paper, we exploit the circuit model in order to investigate some of the fundamental problems. First, we discuss the relation between negative group delay and superluminarity, and then the approximate realization of (positive and negative) delays by lumped systems. Then we consider the spectral condition imposed on input pulses and describe the design of low-pass filters for pulse preparation. Next, in order to increase the advancement, a number of negative-delay circuits are cascaded. We find that an advancement as large as the pulsewidth is possible but the slow increase of the advancement and the exponential increase of out-of-band gain almost prohibit the achievement of further advancements. Finally, by regarding our system as a communication channel, we discuss the causality in lumped systems.

\section{Negative Delay and Superluminal Propagation}

The group velocity $v_{g}$ in a dispersive medium is defined as

$$
v_{g}^{-1}=\left.\frac{d k}{d \omega}\right|_{\omega_{0}}
$$

where the wavenumber $k(\omega)$ is a function of frequency $\omega$. It corresponds to the propagation speed of an envelope of signal whose spectrum is limited within a short interval containing $\omega_{0}$. Similarly, the group delay is defined as

$$
t_{d}=-\left.\frac{d \phi}{d \omega}\right|_{\omega_{0}}
$$

where $\phi(\omega)$ represents the frequency-dependent phase shift. It corresponds to the temporal shift of the envelope of the bandlimited signal passing through a system. For a medium with 
length $L$, the phase shift is given by $\phi(\omega)=-k(\omega) L$ and we have

$$
t_{d}=\frac{d(k L)}{d \omega}=\frac{d k}{d \omega} L=v_{g}^{-1} L .
$$

These two quantities seem almost identical, but the group delay is a more general concept because it can be defined even for a lumped system. The lumped system is a system whose size $L$ is much smaller than the wavelength $2 \pi / k$ of interest. Neglecting the propagation effects, the behavior of the system can be described by a set of ordinary differential equations with respect to time. For distributed systems, on the other hand, the spatio-temporal partial differential equation must be used.

The relation between negative group delays and superluminality can easily be understood when we consider a system consisting of a vacuum path (length $L$ ) and a lumped system (delay $t_{d}$ ) which is located at the end of the path. The total time $t_{\text {total }}$ required for a pulse to pass through the system is

$$
t_{\text {total }}=\frac{L}{c}+t_{d}
$$

The corresponding velocity $v_{g}=L / t_{\text {total }}$ satisfies the relation

$$
\frac{1}{v_{g}}=\frac{1}{c}+\frac{t_{d}}{L} \text {. }
$$

For $t_{d}>0$ (positive delay), $v_{g}$ is smaller than $c$. For $t_{d}<0$ (negative delay), there are two cases. In the case $\left(-t_{d}\right)<L / c$, $v_{g}$ is larger than $c$ (superluminal in a narrow sense), while in the case $\left(-t_{d}\right)>L / c, v_{g}$ becomes negative (negative group velocity). In the latter case, the contribution of the lumped part dominates that of the free propagation path.

Normally, the superluminality has been considered as a propagation effect. But in many cases, it seems more appropriate to discuss in term of the negative group delay for a lumped system. Let us take experimental parameters from [9], in which the negative delay of $t_{d}=-60 \mathrm{~ns}$ was observed. We note that the pulse length $c T_{w}=c \times 2 \mu \mathrm{s}=600 \mathrm{~m}$ is much longer than the cell length $L=6 \mathrm{~cm}$. Therefore, we can safely use the lumped approximation. Since we can eliminate the carrier frequency by the slowly varying envelope approximation, the wavelength of light $(\sim \mu \mathrm{m})$ will not come into play any more.

It should be stressed that the cases where the second term in (4) or (5), which is positive or negative, dominates the first term are very likely. For typical atomic experiments, the bandwidth $\Delta \omega$, which is of the order of megahertz to gigahertz, roughly determines advancement as $\left|t_{d}\right| \sim \Delta n k_{0} L / \Delta \omega \sim 1 / \Delta \omega$, microseconds to nanoseconds, while the passage time $L / c$ is less than the order of nanoseconds. Forcible assignment of a velocity to such cases, $v_{g}=-(1 / 300) c$ in the above example, would have caused some confusion.

\section{GROUP DELAYS-IDEAL AND APPROXIMATE}

\section{A. Mathematical Representation}

A mathematical representation for ideal delays can be written as

$$
v_{\text {out }}(t)=\left(h * v_{\text {in }}\right)(t)=v_{\text {in }}\left(t-t_{d}\right)
$$

where $h(t)=\delta\left(t-t_{d}\right)$ is the impulse response of the system and $t_{d}$ is the delay time. Its Fourier transform is given by

$$
\tilde{V}_{\text {out }}(\omega)=H_{D}(\omega) \tilde{V}_{\text {in }}(\omega)
$$

with

$$
H_{D}(\omega) \equiv \int_{-\infty}^{\infty} d t h(t) e^{-i \omega t}=\exp \left(-i \omega t_{d}\right) .
$$

For $t_{d}>0$ (positive delay), the impulse response $h(t)$ is causal, i.e., it is zero for $t<0$. The positive delay can be realized easily, if you have an appropriate space $\left(L=c t_{d}\right)$. But there is no way to make ideal, unconditional negative delays, because $h(t)$ is noncausal in this case.

\section{B. Building Blocks}

It should be noted that no lumped systems $(L=0)$ can produce ideal positive or negative delays. From now on, let us consider how to make approximate delays with lumped systems. The amplitude and the phase of the ideal response function $H_{D}(\omega)$ are

$$
\begin{aligned}
A_{D}(\omega) & \equiv\left|H_{D}(\omega)\right|=1 \\
\phi_{D}(\omega) & \equiv \arg H_{D}(\omega)=-t_{d} \omega .
\end{aligned}
$$

In [11], we used a circuit having a transfer function

$$
H(\omega)=1+i \omega T
$$

by which negative delay is provided for baseband pulses $\left(\omega_{0}=\right.$ $0)$. Here, we will examine several transfer functions which can be realized with an operational amplifier and a few passive components.

First, we consider a function with a single pole

$$
\begin{aligned}
H_{L}(\omega) & =\frac{1}{1+i \omega T} \\
A_{L}(\omega) & =\frac{1}{\sqrt{1+(\omega T)^{2}}} \sim 1-\frac{(\omega T)^{2}}{2} \\
\phi_{L}(\omega) & =-\tan ^{-1} \omega T \sim-T \omega .
\end{aligned}
$$

The stability condition that all the poles reside in the upper half plane requires $T>0$, therefore, only positive delays $t_{d}=T>$ 0 can be achieved with this type of transfer function. An example of the circuit is shown in Fig. 1(a). Only in the region $|\omega|<1 / T$, the amplitude response is flat and the phase response is linear. The circuit works only for band-limited signals.

Second, we will check a function with a single zero

$$
\begin{aligned}
H(\omega) & =1+i \omega T \\
A(\omega) & =\sqrt{1+(\omega T)^{2}} \sim 1+\frac{(\omega T)^{2}}{2} \\
\phi(\omega) & =\tan ^{-1} \omega T \sim T \omega .
\end{aligned}
$$

In this case, no sign restrictions are imposed on $T$, therefore, both positive and negative delays can be realized; $t_{d}=-T$. A circuit for $H(\omega)(T>0)$ is shown in Fig. 1(b). Perhaps this is the most simple circuit which provides negative delay. Again it works only in the region $|\omega|<1 / T$. Even worse is the rising of gain $A(\omega)$ at the outside of the band. We can also construct 


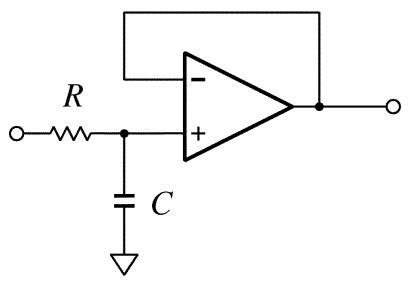

(a)

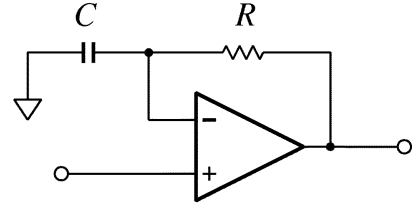

(b)

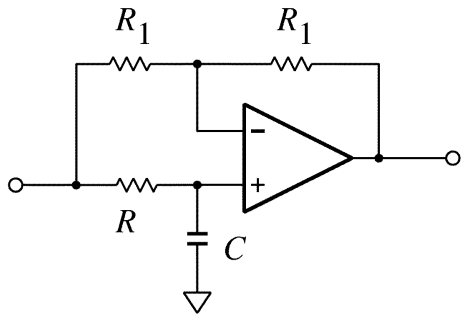

(c)

Fig. 1. Elementary circuits used in negative group-delay experiments. (a) A first order low-pass filter. A series of low-pass filters are used for pulse preparation. (b) Negative group-delay circuit. This simple circuit provides time-advancement for baseband pulses. (c) All-pass filter.

a positive delay circuit utilizing the relation $2-H(\omega)=1+$ $i \omega(-T)$.

By observing the sign restrictions for $H_{L}(\omega)$ and $H(\omega)$, we notice that an asymmetry between the positive and negative delays exists even in lumped systems.

Another interesting transfer function is

$$
\begin{aligned}
H_{A}(\omega) & =\frac{1-i \omega T}{1+i \omega T} \\
A_{A}(\omega) & =1 \\
\phi_{A}(\omega) & =-2 \tan ^{-1} \omega T \sim-2 T \omega
\end{aligned}
$$

which can be realized by the circuit shown in Fig. 1(c). This circuit is called the all-pass filter. The phase function is the same as the above cases aside from the factor 2, but the amplitude response is independent of the frequency as in the case of ideal delay. The stability condition implies $T>0$, therefore, only positive delays are possible.

\section{BANDWIDTH AND DISTORTION}

\section{A. Bandwidth of Negative-Delay Circuit}

It turned out that lumped circuits can provide a delay, positive or negative, only for a band-limited signal. From the approximate transfer function $H(\omega)=1+i \omega T$ for negative delays, we have the imperfect amplitude and phase functions

$$
\begin{aligned}
& A(\omega)=1+O\left(\omega^{2} T^{2}\right) \\
& \phi(\omega)=\omega T+O\left(\omega^{3} T^{3}\right)
\end{aligned}
$$

which are plotted in Fig. 2. We see that the inputs must satisfy the spectral condition

$$
|\omega|<\frac{1}{T}
$$

Otherwise, the output waveform will be distorted due to the higher order terms in (21) and (22). In electronic circuits, a rect-

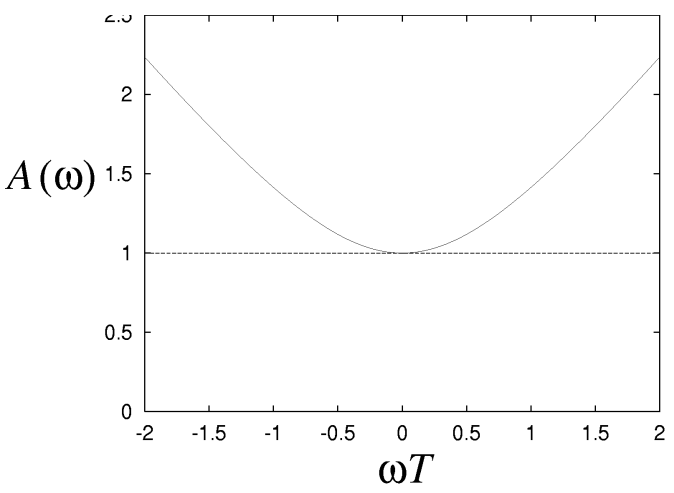

(a)

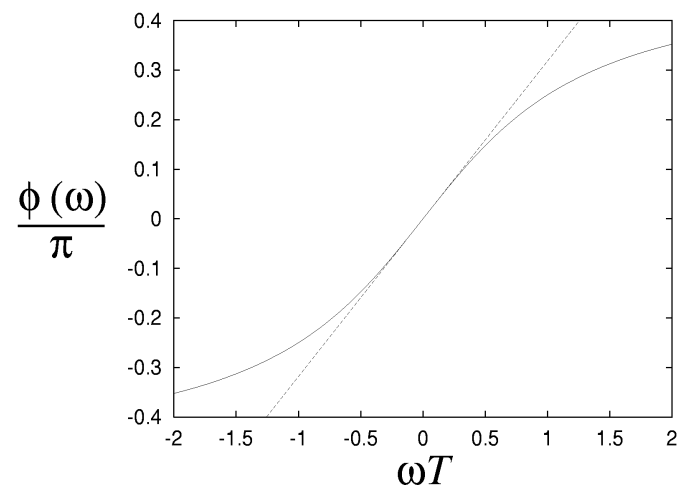

(b)

Fig. 2. Frequency response of approximate negative delay. (a) Amplitude. (b) Phase responses. The response for ideal negative delay are plotted with dashed lines. The approximation is valid only for $|\omega|<1 / T$.

angular pulse is most easily generated. But its spectrum has a long tail; $\sim 1 /|\omega|$. The tail must be suppressed with low-pass filters. The cutoff frequency $1 / T_{L}$ must be smaller than $1 / T$.

\section{B. Low-Pass Filter for Pulse Preparation}

A simple method is to cascade suitable numbers of first-order low-pass filters, whose transfer function is represented by (14), as

$$
H_{L}^{(m)}(\omega)=\frac{1}{\left(1+i \omega \alpha_{m} T_{L}\right)^{m}}
$$

where $\alpha_{m}=\sqrt{2^{1 / m}-1}$ is a normalization parameter to keep the $3-\mathrm{dB}$ cutoff frequency constant. It is reduced as $m$ is increased. Otherwise, due to the decrease of bandwidth, the pulsewidth is broadened and the pulse height is reduced. For better low-pass characteristics, we can use Bessel filters [12], [13], whose transfer function is given by

$$
H_{B}^{(m)}(\omega)=\frac{1}{y_{m}\left(i \omega \alpha_{m} T_{L}\right)}
$$

where $y_{m}(x)$ is the $m$ th Bessel polynomials and the parameter $\alpha_{m}$ is determined so that $H_{B}^{(m)}\left(T_{L}^{-1}\right)=1 / \sqrt{2}$.

The effect of filtering is shown in Figs. 3 and 4. The initial, rectangular pulse $U(t)-U\left(t-T_{w}\right)$ is sent to a series of filters, where $U(t)$ represents a unit step function. As the order $m$ of the filter is increased, the high-frequency tails are more suppressed, and accordingly, the waveform becomes smoother. Exceeding a few stages, the waveforms look very similar to each 


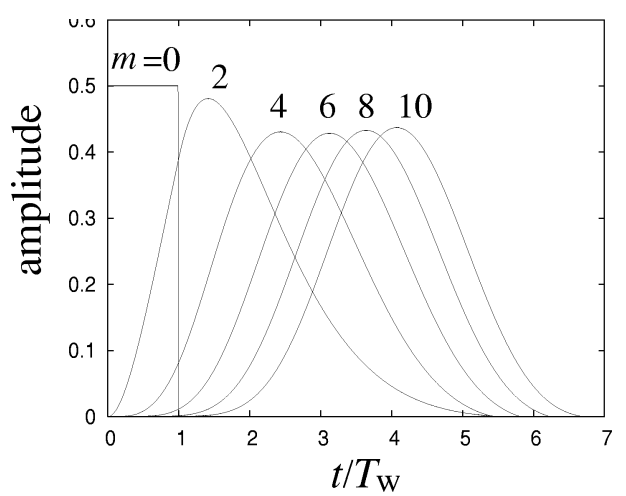

Fig. 3. Rectangular pulse of width $T_{w}\left(=T_{L}\right)$ is filtered by $m$ th-order Bessel filter with cutoff frequency $1 / T_{L}$. The height of the original pulse $(m=0)$ is unity but it is halved in the graph.

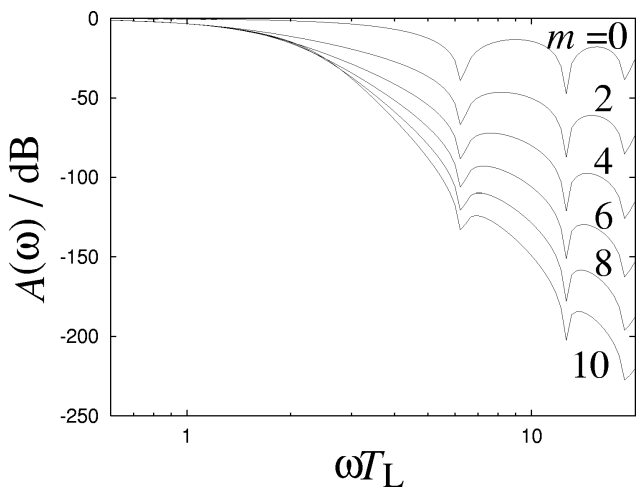

Fig. 4. Spectra of low-pass-filtered pulses (log-log plot). The high-frequency components strongly suppressed as $m$ increases.

other. But the leading edge scales $\sim t^{m}$ and the peak position is delayed. The delay, which is due to the phase transfer function of the low-pass filters, is unavoidable. We will see in Section VI that $m$ must be increased in order to attain large advancement. The pulsewidth approaches a value determined by the cutoff frequency $T_{L}^{-1}$, if the initial pulsewidth is smaller than $T_{L}$.

From the way of preparation of input pulse with low-pass filters, we can interpret that the leading edge is shaped so as to be more predictable. The future can well be predicted, if enough restrictions are imposed on the pulse.

\section{Gaussian Pulse}

The Gaussian pulse is widely used in negative delay or superluminal propagation experiments. It is because of the rapid tail off of the spectrum and its mathematical simplicity. But there seems no natural implementation which generates a Gaussian pulse. It is usually synthesized numerically and the calculated data is fed to a digital-to-analog converter. It should be noted that the ideal Gaussian pulse has a infinitely long leading edge. What we can generate practically is a truncated Gaussian pulse

$$
v_{I}(t)=u(t) g\left(t-t_{0}\right)
$$

where $g(t)=\exp \left(-t^{2} / 2 T_{w}^{2}\right)$. A causal function $u(t)$ is a unit step function or a smoothed version of it. This truncation necessarily introduces discontinuities for $d^{k} v_{I} / d t^{k}(k=0,1, \ldots)$
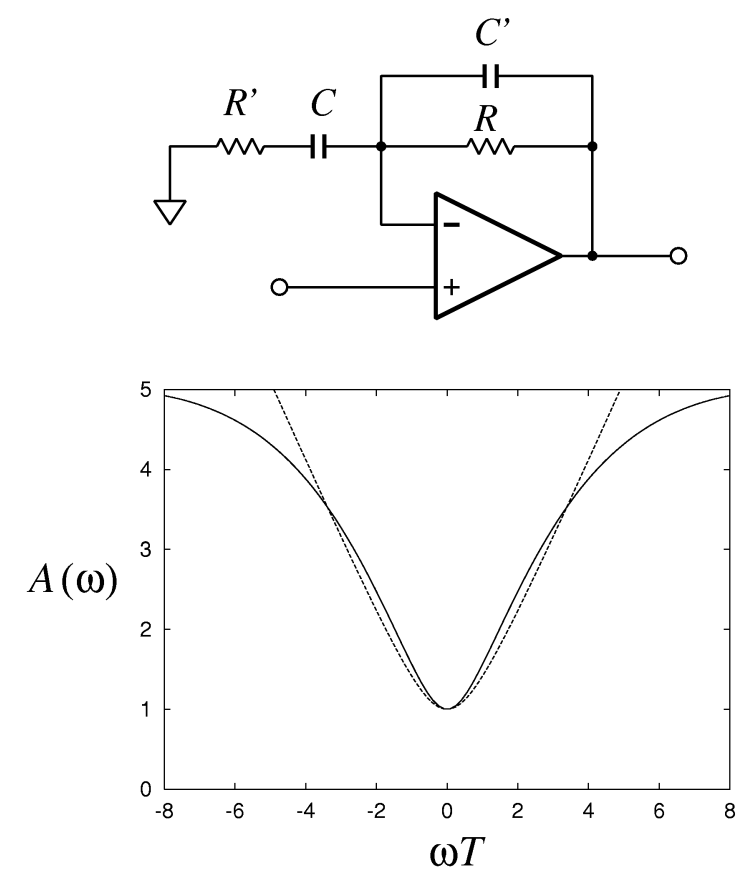

(b)

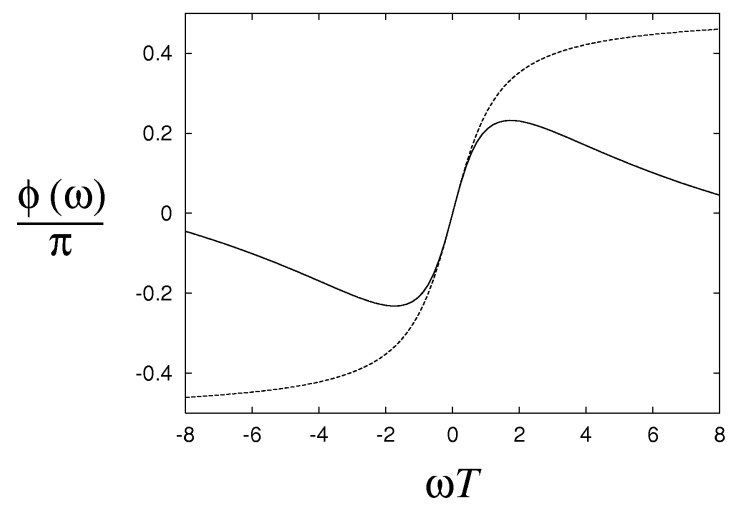

(c)

Fig. 5. (a) Circuit diagram of a practical negative-delay circuit. Gain divergence for higher frequency is suppressed by $C^{\prime}$ and $R^{\prime}$. (b) Amplitude response function $A(\omega)$. (c) Phase response function $\phi(\omega)$. Parameters are $a=C^{\prime} / C=0.2$, are $b=R^{\prime} / R=0.05$. The case of $a=b=0$ is also plotted. Usable frequency region is limited within $|\omega| T<1$.

at $t=0$ and associates high-frequency components. However small they may look like, they will eventually be revealed by a negative-delay circuit with advancement larger than $t_{0}$. In order to reduce the effect of truncation, $t_{0}$ must be increased, which corresponds to the increase of $m$ in the pulse preparation with low-pass filters.

\section{EXPERIMENT}

\section{A. Practical Design}

Fig. 5(a) shows a practical circuit for negative delays [11]. The components $C^{\prime}$ are $R^{\prime}$ are added to the circuit of Fig. 1(b) in order to suppress the gain for higher frequency. Its transfer function can easily be derived. First, we see that

$$
\tilde{V}_{-}=\frac{Z_{C}}{Z_{C}+Z_{R}} \tilde{V}_{\text {out }}
$$




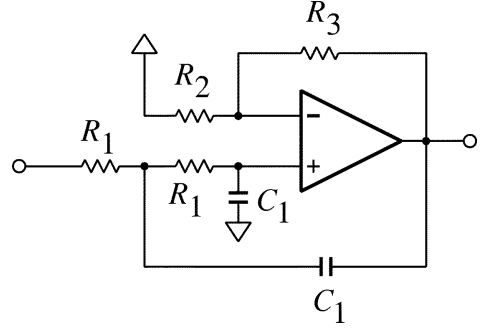

Fig. 6. Second-order Bessel low-pass filter.

where $Z_{C}=R^{\prime}+1 / i \omega C, Z_{R}=\left(R^{-1}+i \omega C^{\prime}\right)^{-1}$. If we assume a large gain of the operational amplifier, the virtual short condition $\tilde{V}_{\text {in }} \sim \tilde{V}_{-}$holds and we have

$$
\begin{aligned}
H_{N}(\omega) & =\frac{\tilde{V}_{\text {out }}}{\tilde{V}_{\text {in }}} \\
& =\left(1+\frac{Z_{R}}{Z_{C}}\right) \\
& =1+\frac{i \omega T}{(1+i \omega a T)(1+i \omega b T)}
\end{aligned}
$$

where $T=C R$. If $a \equiv C^{\prime} / C \ll 1$ and $b \equiv R^{\prime} / R \ll 1$ are satisfied, then the transfer function is approximated as $H_{N}(\omega) \sim$ $1+i \omega T$ near the origin $(|\omega|<1 / T)$. Thanks to $a$ or $b$, the maximum gain is limited by $1 /(a+b)$. The response functions for $a=0.2, b=0.05$ are plotted in Fig. 5(b) and (c). The phase slope at the origin is almost conserved but the usable bandwidth is reduced.

Second-order Bessel filters are used in the experiment, because the second-order filter can be realized with an operational amplifier [12], [13]. The circuit diagram is shown in Fig. 6. The frequency response is

$$
H_{B}^{(2)}(\omega)=\frac{1}{1+i \omega \alpha_{2} T_{L}+\frac{\left(i \omega \alpha_{2} T_{L}\right)^{2}}{3}}
$$

where $T_{L}=1.272 R_{1} C_{1}$ is the inverse of cutoff frequency and $R_{3} / R_{2}=0.268$.

\section{B. Experimental Result}

In Fig. 7, we show the overall block diagram for the negative-delay experiment. The complete circuit diagram is presented in [11]. The pulse generator on the top is composed of a single-shot pulse and two second-order Bessel filters. Triggered by the switch, a timer integrated circuit (ICM7555) generates a rectangular pulse with duration $1.5 \mathrm{~s}$. The pulse is shaped by the filters. The cutoff frequency is chosen as $\omega_{c}=1 / T_{L}=0.35 / T$, so that $A(\omega)$ and $\phi(\omega)$ can be considered to be constant and linear, respectively. $T=C R$ is the time constant of the negative-delay circuits.

Two negative-delay circuits are cascaded for larger advancement. The circuit parameters are $R=1 \mathrm{M} \Omega, C=0.22 \mu \mathrm{F}$, $a=0.1$, and $b=0.01$.

The input and output terminals are monitored by LEDs. Their turn-on voltage is about $1.1 \mathrm{~V}$.

The experimental result is shown in Fig. 8. The input and output waveforms are recorded with an oscilloscope. The time

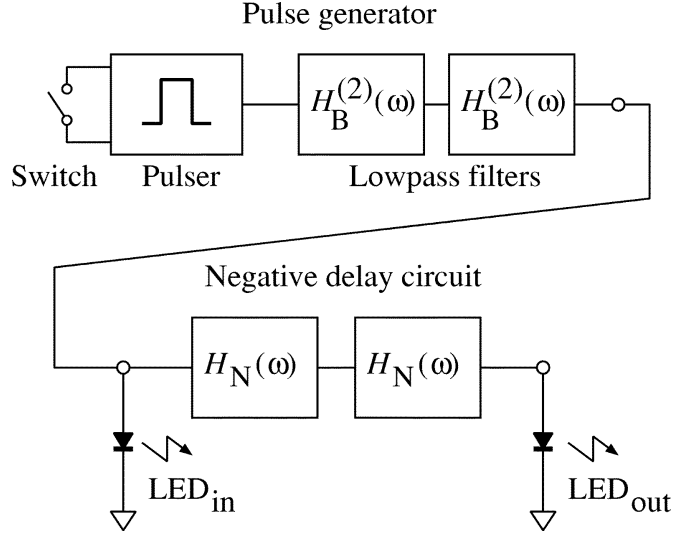

Fig. 7. Experimental setup.

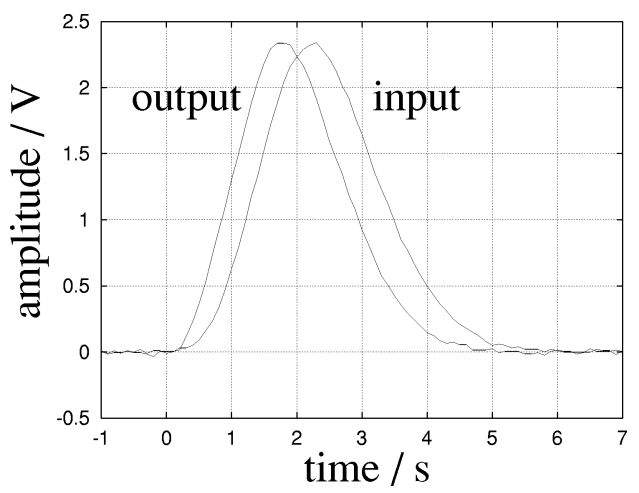

Fig. 8. Experimental result. The traces of oscilloscope show the input and output pulses. Pulse is advanced about $0.5 \mathrm{~s}$.

origin $(t=0)$ is the moment when the switch is turned ON or the rising edge of the initial rectangular pulse.

We see that the output pulse precedes the input pulse considerably (more than $20 \%$ of the pulsewidth). The slight distortion of the output waveform is caused by the nonideal frequency dependence of $A(\omega)$ and $\phi(\omega)$.

The observed advancement of $\sim 0.5 \mathrm{~s}$ agrees well with the expected value $2 T=2 C R=0.44 \mathrm{~s}$.

General purpose operational amplifiers (TL082) are used for low-pass filters and negative-delay circuits. The time scale has been chosen so that we can directly observe the negative delay with two LEDs connected at the input and the output terminals. The whole experimental setup can be battery operated and self contained. If we want to observe the waveform, instead of oscilloscopes, we can use two analog voltmeters (or circuit testers) to monitor the waveforms.

\section{CASCADING-FOR LARGER ADVANCEMENT}

\section{A. Normalized Advancement}

We have seen that in typical situations the advancement $\left(-t_{d}\right)$ is fairly smaller than the pulsewidth $T_{w}$. Typically, the relative advancement $r \equiv\left|t_{d}\right| / T_{w}$ only reaches to a few percents.

To see the reason, we consider a Gaussian pulse $v(t)=V_{0} \exp \left(-t^{2} / 2 T_{w}^{2}\right)$ and its Fourier transform $\tilde{V}(\omega)=\sqrt{2 \pi} T_{w} \exp \left(-\omega^{2} T_{w}^{2} / 2\right)$. When it is passed through the negative-delay circuit, the power is amplified owing to the 
rising amplitude response (16). We define the excess power gain $\eta$ as

$$
1+\eta=\frac{\int_{-\infty}^{\infty}|H(\omega) \tilde{V}(\omega)|^{2} d \omega}{\int_{-\infty}^{\infty}|\tilde{V}(\omega)|^{2} d \omega}
$$

which can be used as a measure of distortion. Now we have a relation between $\eta$ and the relative advancement $r$

$$
\eta=\frac{\int_{-\infty}^{\infty} \omega^{2} T^{2} e^{-\omega^{2} T_{w}^{2} / 2} d \omega}{\int_{-\infty}^{\infty} e^{-\omega^{2} T_{w}^{2} / 2} d \omega}=2\left(\frac{T}{T_{w}}\right)^{2}=2 r^{2} .
$$

For example, if we allow $\eta=5 \%$, then the relative advancement is $r=15 \%$ at most.

If we want to increase the advancement $\left|t_{d}\right|$, for a given system, the bandwidth must be reduced, which results in the increase of pulsewidth $T_{w}$, and $r$ does not increase.

\section{B. Degression of Time Constant}

We will try to increase the relative advancement by cascading the negative group-delay circuits in series. The transfer function for $n$ stages

$$
\begin{aligned}
H^{(n)}(\omega) & =(1+i \omega T)^{n} \\
A^{(n)}(\omega) & \sim 1+\frac{(\sqrt{n} \omega T)^{2}}{2} \\
\phi^{(n)}(\omega) & \sim n \omega T .
\end{aligned}
$$

At first, we may expect that the total advancement increases in proportion to $n$ because the slope of $\phi^{(n)}(\omega)$ at the origin increases as $n T$. But we should notice by looking at $A^{(n)}(\omega)$ that the usable bandwidth is reduced as $n$ increased for $T$ fixed. In other words, for a given input pulsewidth $T_{w}, T$ must be reduced as $T_{w} / \sqrt{n}$. Thus, the total advancement scales as

$$
\left(-t_{d}\right) \propto n \times\left(\frac{T_{w}}{\sqrt{n}}\right)=\sqrt{n} T_{w} .
$$

It should be noted that the gain outside of the band is increases very rapidly. Spectral tails of the input pulse must be suppressed enough. Otherwise, they could be amplified to distort the pulse shape. From the asymptotic forms of $H^{(n)}(\omega)$ and $H_{L}^{(m)}(\omega)$, we see that the condition $m>n$ must be satisfied. Order $m$ of low-pass filters must be increased cooperatively. It is possible to increase the advancement as large as the pulsewidth or more, but the advancement increases very slowly; $1 / \sqrt{n}$. Fig. 9(a) represents an example of simple-minded cascading, where $T$ is kept constant. The waveforms are rapidly distorted as $n$ increased.

Fig. 9(b) shows the $1 / \sqrt{n}$ cascading, where $T$ is reduced as $T / \sqrt{n}$. The pulse shape is preserved fairly well. The input pulse is filtered with five tenth-order Bessel filters $(m=50)$. Fig. 10 shows the case of $n>m$. The input rectangular pulse is filtered by a fourth-order Bessel filter $(m=4)$. For $n=4$ cascading, scars of the initial pulse appear at $t=0$ and $t=T_{w}$, and they become more prominent as $n$ increases.

\section{Out-of-Band Gain}

As we have seen, the out-of-band gain is the primary obstacle toward large advancement. In order to estimate how the gain
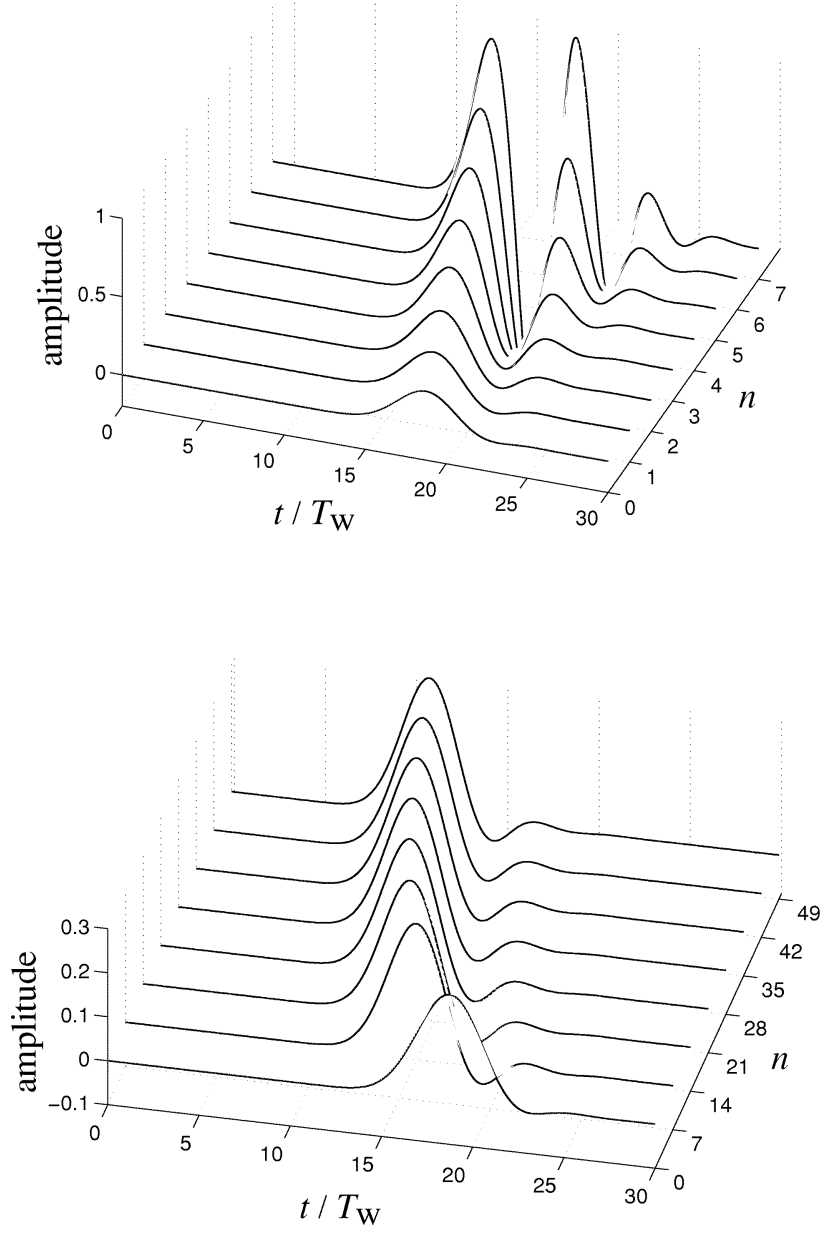

(b)

Fig. 9. Cascading of negative-delay circuits. The input pulse is filtered by a series of five tenth-order Bessel filters $(m=50)$. (a) Simple cascading with fixed time constant. The pulse is forwarded further as the number of circuits $n$ increases, but the waveform is heavily distorted after a few stages. (b) Cascading with reduced time constant as $1 / \sqrt{n}$. Owing to small distortion, we can increase $n$ even though the advancement per stage is small. With $n=49$ stages, the advancement clearly larger than the pulsewidth is achieved.

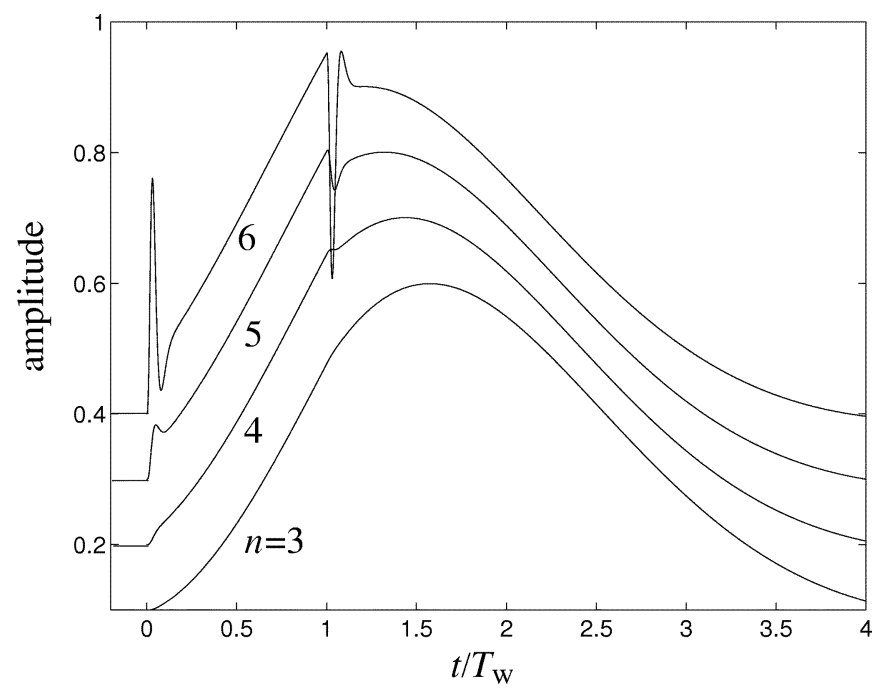

Fig. 10. Breakdown of cascading. The input pulse is filtered by a fourth-order Bessel filter $(m=4)$. We see that, for $n>m$, the pulse advancement halts. Especially at $t=0$ and $t=T_{w}$, which correspond to the turn-on and the turn-off of the original rectangular pulse, respectively, strong deformation is observed. Curves are offset for clarity. 


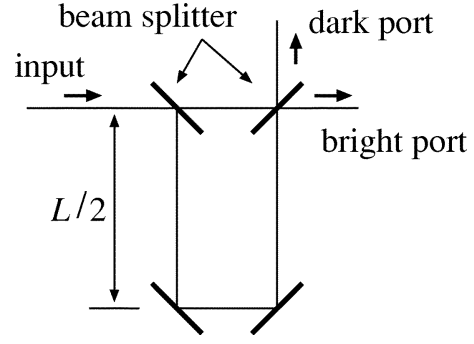

Fig. 11. Mach-Zehnder interferometer as a negative group-delay device. The reflectivity of two beam splitters is slightly smaller than $50 \%$. The output from dark port is advanced.

increases, we use a realistic transfer function (28), which has a finite maximum gain $A_{\max } \sim 1+(a+b)^{-1}$. For $a=0.2$, $b \ll a, n=50$, we have

$$
A_{\text {max }}^{n}=6^{50} \sim 10^{40} .
$$

Such a huge gain will certainly induce instabilities. The noise level must also be suppressed. If we increase $a$ or $b$ to reduce $A_{\max }$, then the bandwidth is significantly reduced as seen in Fig. 5. For the reduced bandwidth, we have to increase the pulsewidth $T_{w}$, which diminishes the relative advancement $\left|t_{d}\right| / T_{w}$.

We see from this example, a large negative delay comparable to the pulsewidth is very hard to achieve or almost prohibitive. The allowable gain would be limited by system-dependent factors such as a performance of active devices, a threshold for instabilities, fluctuation due to quantum noise, and so on.

Again we notice the asymmetry between the negative and the positive delays. For positive delays, gain problem does not occur. In fact, the cascading of low-pass filters yields a large amount of delay without difficulty.

\section{DISCUSSION}

\section{A. Interference in the Time Domain}

From the minimal transfer function $H(\omega)=1+i \omega T$ for the negative delay, we see that in time domain the input-output relation can be written as

$$
v_{\text {out }}(t)=\left(1+T \frac{d}{d t}\right) v_{\text {in }}(t)=v_{\text {in }}(t)+T \frac{d v_{\text {in }}}{d t}(t) .
$$

Here, the two terms interfere constructively at the leading edge and destructively at the trailing edge. The addition of the time derivative to the original pulse results in the pulse forwarding.

This time-domain picture is useful to devise a new system which shows negative delays. The Mach-Zehnder interferometer shown in Fig. 11 is such an example. First, we assume $R=$ $1 / 2$ for the reflectivity of the beam splitters. The path difference $L$ is chosen so as to satisfy the condition $\lambda \ll L=c \tau \ll c T_{w}$, where $\lambda$ is the wavelength, $T_{w}$ is the pulsewidth, and $\tau=L / c$ is the delay time due to the path difference. We can tune the path length so that the transmission for one port is unity. Then for the steady state, there appears no output at the other port (dark port) owing to the destructive interference.

For time-dependent inputs, however, the cancellation is incomplete and the output corresponding to the time derivative of

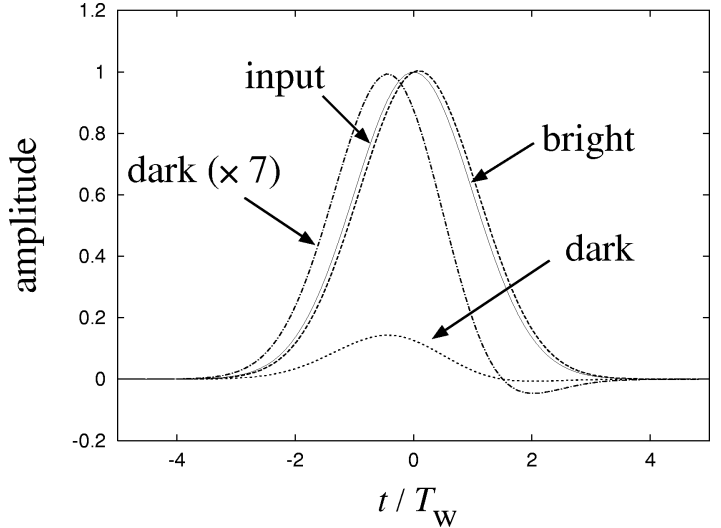

Fig. 12. Pulse advancement by Mach-Zehnder interferometer. A Gaussian input pulse $g(t)=\exp \left(-t^{2} / 2 T_{w}^{2}\right)$ is used. The parameters are $\tau=0.17 T_{w}$, and $\epsilon=0.06$.

the amplitude appears at the dark port (see Fig. 12). If we superpose this output with the original waveform, we will have the advancement as shown in (37). The superposition can easily be provided by unbalancing the amplitude of each path. We set the reflectivity of the two beam splitters as slightly smaller than $50 \%: R=1 / 2-\epsilon$. Then the output of the dark port becomes

$$
\begin{aligned}
\tilde{E}_{\text {dark }}(t) & =(1-R) \tilde{E}_{\text {in }}(t)-R \tilde{E}_{\text {in }}(t-\tau) \\
& \sim 2 \epsilon\left(1+\frac{\tau}{4 \epsilon} \frac{d}{d t}\right) \tilde{E}_{\text {in }}(t)
\end{aligned}
$$

and the advancement of $\tau / 4 \epsilon$ is achieved. $\tilde{E}_{\text {in }}(t)$ and $\tilde{E}_{\text {dark }}(t)$ are the envelope of the input field and the dark port field, respectively. The usable bandwidth of the system is $\Delta \omega \sim 1 / \tau$, for which the darkness of the dark port is ensured.

This is an example of all-passive systems with negative delay. It should be noted that when we increase the advancement by decreasing $\epsilon$, the transmission is decreased accordingly. It is also true for the superluminal propagation of evanescent waves and tunneling waves [4], [5].

This model convinces us that the negative group delay and the superluminal group velocity are the simple consequence of wave interference.

\section{B. Causality and Negative Delay}

It has been well recognized and confirmed in many ways that the front velocity is connected with the causality, and the causality has no direct connection with the group velocity. But one is still apt to connect the group velocity with the causality because many practical communication systems utilize pulse modulation to send information.

Let us examine our system shown in Fig. 13 in terms of causality. By pushing the switch $O$, a rectangular pulse $A$ is generated. Feeding it into the low-pass filter, a band-limited pulse $B$ is prepared. Finally, sending it through the negative-delay circuit, an advanced pulse $C$ is created. This would be a cause-effect chain in a casual sense. However, the reversal of the chronological order between $B$ and $C$ causes trouble for the naive picture. One may think that making use of this twist, it is possible to send information to the past despite of the causality. Of course this is wrong. 


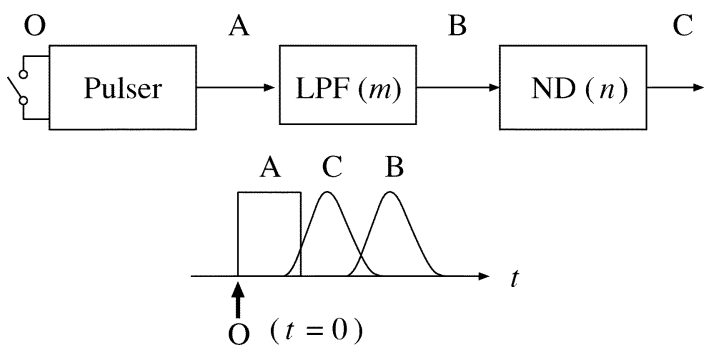

Fig. 13. Communication channel with negative group delay. Inset shows timing of pulses at each site.

First, we should realize that in a strict sense, all the pulses $A$, $B$, and $C$ are causal to each other because they are the signals in a single lumped system. When an impulse is applied to a quiescent lumped system, then all parts respond instantaneously. Some of them look delayed, but they just started smoothly as $t^{k}$ $(k>1)$. All the pulse fronts share the time $t=0$, when the switch is turned on $O$. Therefore, the above discussion on the order is totally pointless.

But one may still shelve the theory, which deals with the almost unseen signals just after $t=0$, considering practical situations where the information is related to the peak position or the rising edge where a half of the pulse peak is reached. However, in order to generate a smooth pulse $B$ which is acceptable to the negative-delay circuit, we have to make a decision well in advance (before $t=0$, in this case) because of the delay caused by the low-pass filter. Once we miss the timing, the number $m$ of the low-pass filters must be reduced in order to catch up. But breaking the condition $m>n$, the pulse cannot be forwarded any more and is distorted badly.

Let us regard the negative-delay circuit of Fig. 13 as a communication channel. We assign three people, Alice, Bob, and Clare, on the sites $A, B$, and $C$, respectively. Clare always finds a pulse before Bob does, i.e., she can always predict Bob's pulse. But Bob has no control over his pulses; he cannot cancel a pulse initiated by Alice. The real sender of the pulse is not Bob, but Alice. Bob is just an observer standing at the sending site. This scenario tells us that comparing the input and the output pulses of superluminal channel is somewhat nonsense and that the real start point of the input pulse should be considered (see Section IV-C).

\section{CONCLUDING REMARKS}

The negative group delay is already utilized in many practical applications implicitly. Signals from slow sensors, such as a hot-wire anemometer, are compensated by a differentiator with a transfer function $1+i \omega T$. In proportional, integral, and derivative (PID) controllers the derivative element $D$ is used to predict the behavior of the system and to improve the dynamic response. When a capacitive load is connected to the output of an operational amplifier, an additional feedback loop with derivative element is used, which is called lead compensations. All these efforts are to compensate delays in a system as far as possible but the excessive use will result in instabilities or noise problems.

We have explored many aspects of negative delays and superluminality utilizing circuit models. The use of circuit models is very helpful because the choice of parameters are very flexible and many handy circuit-simulation software are available. Extension to nonlinear cases and to distributed systems [14] will be very interesting.

\section{ACKNOWLEDGMENT}

M. Kitano would like to thank R. Chiao and all the participants of the mini program on Quantum Optics at the Institute of Theoretical Physics, University of California, Santa Barbara, for stimulating and inspiring discussions. He would also like to thank K. Shimoda for information about practical use of negative-delay circuits.

\section{REFERENCES}

[1] L. Brillouin, Wave Propagation and Group Velocity. New York: Academic, 1960, pp. 113-137.

[2] S. Chu and S. Wong, "Linear pulse propagation in an absorbing medium,” Phys. Rev. Lett., vol. 48, pp. 738-741, Mar. 1982.

[3] R. Y. Chiao and A. M. Steinberg, "Tunneling times and superluminality," Prog. Opt., vol. XXXVII, pp. 345-405, 1997.

[4] G. Enders and G. Nimtz, "On the superluminal barrier traversal," $J$. Phys., vol. I2, pp. 1693-1698, Sept. 1992.

[5] A. M. Steinberg, P. G. Kwiat, and R. Y. Chiao, "Measurement of the single-photon tunneling time," Phys. Rev. Lett., vol. 71, pp. 708-711, Aug. 1993.

[6] M. W. Mitchell and R. Y. Chiao, "Causality and negative group delays in a simple bandpass amplifier," Amer. J. Phys., vol. 66, pp. 14-19, Jan. 1998.

[7] — "Negative group delay and 'fronts' in a causal systems: An experiment with very low frequency bandpass amplifiers," Phys. Lett., vol. A230, pp. 133-138, June 1997.

[8] H. Cao, A. Dogariu, and L. J. Wang, "Negative Group Delay and Pulse Compression in Superluminal Pulse Propagation," unpublished.

[9] L. J. Wang, A. Kuzmich, and A. Dogariu, "Gain-assisted superluminal light propagation," Nature, vol. 406, pp. 277-279, July 2000.

[10] A. Dogariu, A. Kuzmich, and L. J. Wang, "Transparent anomalous dispersion and superluminal light-pulse propagation at a negative group velocity," Phys. Rev. A, vol. 63, pp. 053 806-1-11, Apr. 2001.

[11] T. Nakanishi, K. Sugiyama, and M. Kitano, "Demonstration of negative group delays in a simple electronic circuit," Amer. J. Phys., vol. 70, pp. 1117-1121, Nov. 2002.

[12] U. Tietze and C. Schenk, Electronic Circuits. Berlin, Germany: Springer-Verlag, 1991, pp. 350-408.

[13] P. Horowitz and W. Hill, The Art of Electronics, 2nd ed. Cambridge, U.K.: Cambridge Univ. Press, 1989, pp. 175-284.

[14] R. W. Ziolkowski, "Superluminal transmission of information through an electromagnetic metamaterial," Phys. Rev. E, vol. 63, pp. $046604-$ $1-13$, Apr. 2001

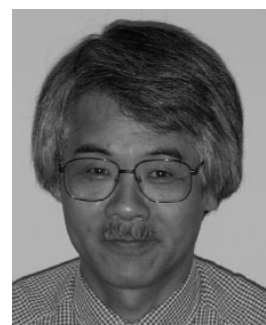

Masao Kitano was born in Kyoto, Japan, on August 5, 1952. He received the B.S., M.S., and Ph.D. degrees in electronic engineering from Kyoto University, Kyoto, Japan, in 1975, 1977, and 1984, respectively.

In 1977, he joined the Department of Electronics, Kyoto University. Since 1999, he has been a Professor at the Department of Electronic Science and Engineering, Kyoto University. He spent the academic years 1984-1986 on leave at the Department of Physics, Princeton University, Princeton, NJ. His research interests include quantum optics, nearfield optics, optical pumping, electromagnetism, and quantum computation.

Dr. Kitano is a member of the American Physical Society, the Institute of the Electronics, Information and Communication Engineers, the Physical Society of Japan, the Laser Society of Japan, and the Japan Society of Applied Physics. 


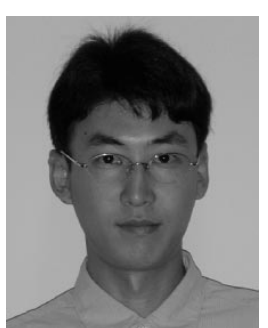

Toshihiro Nakanishi was born in Mie Prefecture, Japan, on June 22, 1976. He received the B.S. and M.S. degrees in electronic science and engineering from Kyoto University, Kyoto, Japan, in 2000 and 2002 , respectively.

$\mathrm{He}$ is currently a graduate student in electronic science and engineering at Kyoto University. His research interests concern quantum Zeno effect, quantum optics, and laser cooling.

Mr. Nakanishi is a member of the Physical Society of Japan.

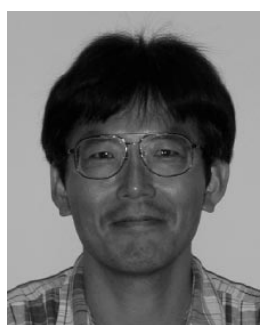

Kazuhiko Sugiyama was born in Aichi Prefecture, Japan, on April 15, 1961. He received the B.E. and M.E. degrees in electronic engineering from Kyoto University, Kyoto, Japan, in 1984 and 1986, respectively. He received the Ph.D. degree in electronic engineering from Kyoto University in 1997, for his work of trapping and laser cooling of ytterbium ions.

From 1986 to 2001, he was engaged in the research of optical frequency metrology at the National Research Laboratory of Metrology, Tsukuba, Japan. In 2001, he joined the Graduate School of Electronic Science and Engineering, Kyoto University, as an Associate Professor. His current research interests are the realization of precise optical clocks and quantum optics.

Dr. Sugiyama is a member of the Physical Society of Japan, the Japan Society of Applied Physics, the Optical Society of Japan, and the Optical Society of America. 